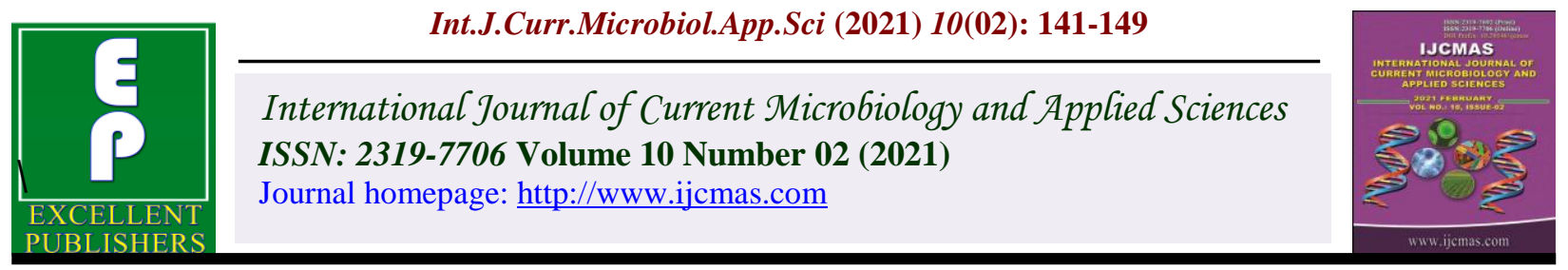

\title{
Microbiome of Groundnut (Arachis hypogaea L.) Rhizosphere Infected with Macrophomina pheseolina Root Rot
}

\author{
M. V. Parakhia* and B. A. Golakia \\ Department of Biotechnology, Junagadh Agricultural University, Junagadh, Gujarat, India \\ *Corresponding author
}

A B S T R A C T

Keywords

Microbiome,

Groundnut,

Arachis hypogaea,

Macrophomina

pheseolina

Article Info

Accepted:

12 January 2021

Available Online:

10 February 2021
This study reports the analyses of the rhizospheric microbiome of the groundnut (Arachis hypogaea L.) from Gujarat, India. Samples were collected using standard protocols and 16S rRNA gene V3-V4 region amplicon sequencing was performed to identify the microbial communities prevalent in the rhizosphere. A total of 9527329 quality checked reads with the average read length $232 \mathrm{bp}$ were analysed using QIIME to reveal the abundance of Acidobacteria. Proteobacteria, and Planctomycetes. The diversity analysis for the community highest 116 spices were observed in infected sample.

\section{Introduction}

In the world where the population keeps increasing at an alarming rate while food is inadequate, it is compulsory to new alternatives to feed the growing population need to be identified. The answer lies in harnessing and exploiting the beneficial interactions between plants and their rhizospheric microbiome to increase plant health and productivity. An understanding of the mechanisms that govern such interactions is essential to increase plant health and productivity.

To amplify the food production over use of conventional chemical pesticides from the years also has resulted in adverse effects on the environment and non-target organisms. The Groundnut \& other crops in India often suffer from various type of root rot \& wilt diseases caused by fungi. Among these the dry root rot caused by Macrophomina phaseolina (Tassi) Goid has been noticed to cause 33.33 per cent seed rotting and 23.80 per cent post emergence mortality (Gupta and Kolte, 1981).

To study the dry root rot disease conventional microbiological methods were used. in these method only cultivable microbes and its interaction can be studied. it is identified that 98\% microbes are not cultivable means cannot grown in laboratory media. it is 
require to study the role of uncultivable microbe's on infection to the plant. The rhizosphere zone around the root is the largest microorganisms ecology in the world. The rhizomicrobiome has the capability to help fight numerous biotic and abiotic stresses as well as enhance plant health and productivity. rhizomicrobiome study of microorganisms cultivable as well as uncultivable to understand the interaction effect of microorganisms on plant productivity and health.

Microbiome is defined as the totality (Cultivable and Uncultivable) of microorganisms and their collective genetic material present in or on the plant or in another environment.

Metagenomics combines the power of genomics, bioinformatics, and systems biology. Operationally, it is novel in that it involves study of the genomes of many organisms simultaneously. It provides new access to the microbial world; the vast majority of microbes cannot be grown in the laboratory and therefore cannot be studied with the classical methods of microbiology. Although community ecology is not new to microbiology, the ability to bring to bear the power of genomics in the study of communities initiates an unparallel opportunity.

Normally the plant associated microbes are studied by culture based methods. Unfortunately this culture based methods could not represent the actual microbial composition of the environment. Culture based methods are, most often, known to have bias towards few bacterial groups. Microbes that cannot grow on artificial media can study using direct nucleic acid extracted from samples. In the recent times high-throughput technologies are developed to expose the interactions between complex microbial communities and their hosts. For prokaryotes, sequencing of $16 \mathrm{~S}$ rRNA gene is the most common method used for the identification of different species and strains. By analyzing on $16 \mathrm{~S}$ rRNA gene region, it is possible to taxonomically group different bacteria.

Along with increasing plant productivity, the soil microbiome also provides an important role in disease-suppressive soils. The ability of a soil to suppress disease is of key importance in measuring soil productivity (Janvier et al., 2007). There are many PGPRs that aid in disease suppression via the release of antimicrobial or antifungal compounds that deter plant pathogens (Garbeva et al., 2004; Weller et al., 2002).

Elevated levels in species richness and diversity produce high functional redundancy within the soil microbiome, allowing it to quickly recover during stress (Nannipieri et al., 2003; Yin et al., 2000). The high functional redundancy in soil microbial diversity also confers protection against soilborne diseases (Brussaard et al., 2007; Garbeva et al., 2004; Mendes et al., 2011; Nannipieri et al., 2003). Consequences of interaction between rhizosphere microbiome, especially the dominant microbial community and dry root rot fungus, Macrophomina have not been systematically analyzed. Unraveling the composition and function of the groundnut rhizosphere microbiome is not only important for our basic understanding of host-microbe interactions but also of great agronomic and ecological importance as well. Analysis of groundnut rhizosphere microbiome is of great commercial importance for groundnut farming as the crop is susceptible to several pathogens. Therefore, there is a need to decipher the microbial composition of groundnut rhizosphere and their influence on economically important diseases like dry root rot. 
With these background a thesis project on "Utilizing groundnut (Arachis hypogaea L.) rhizosphere microbiome for biological control of dry root rot" is envisaged with the following objectives.

\section{Materials and Methods}

\section{Sample collections}

Rhizosphere soil of groundnut variety GG-20 was collected. Samples were collected from selected sites having different type of soils (table no.3.1) with grown GG-20 groundnut variety having healthy and/or dry root rot infected plant for microbiome study.

\section{DNA Extractions and sequencing}

DNA was extracted from all 10 samples using The Power Soil® DNA Isolation Kit with minor modification and accordance to manufacture's instruction by MoBio laboratories Inc. Sample library preparation and sequencing were carry out as per ion torrent $\mathrm{S} 4$ sequencing kit guideline.

\section{Data analysis}

Reads obtained from sequencer were converted to bam file and Fastq format in ion torrent server

\section{Quality control for reads}

Primary quality control by the machine inbuild software to reduce ambiguous reads and final fastq file used for the secondary quality checking using the CLC genomics workbench 20.0. In that CLC processed for the adapter, barcodes, ambiguity and primer, duplicate reads are screened and removed.

\section{Binning process}

Reads are used for mapping with using databases RDP, Greengen, and SILVA for
$16 \mathrm{~S}$ region. Binning process were carry out in MG-RAST (https://www.mg-rast.org/) and QIIME (http://qiime.org/). QIIME software was used for analysis of $16 \mathrm{~S}$ rDNA sequencing data. QIIME is comprehensive software comprising of tools and algorithms such as Fast Tree for heuristic based maximum-likelihood phylogeny inference (Price et al., 2010).

Ion Reporter (https://ionreporter.thermofisher. com/ir/) tool were used to identify the genus or species level of microbes present in complex polybacterial research samples, using both Greengenes and premium MicroSEQ ID 16S rRNA reference databases. Ion Reporter metagenomics workflow: Unaligned binary data files (Binary Alignment Map, BAM) generated by the Ion Torrent Ion S5 were uploaded to Ion Reporter and analysed using default settings. Calls were made by assessing the most specific unique taxonomic level identified using the mapped sequences.

The Ion Reporter metagenomics workflow was developed for primer information, classification information, percent ID, and mapping information. Also identified population diversity for whole metagenome at taxonomic level with the interactive display

\section{Data process and visualisation}

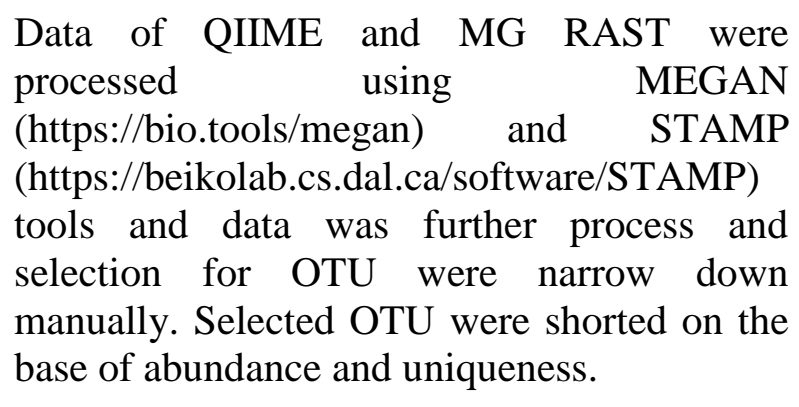

Selected Data/OTU were compared on the basis of sample site variation and status of groundnut (Healthy/Infected). Data also 
analyzed manually for selected genus and species richness in the samples/sites.

\section{Diversity analysis}

Using QIME (Observed-species, Shannon, Simpson and Good-coverage) alpha diversity through 8 indices was analyzed. Beta diversity was also calculated by QIIME (Version 1.9.1) on unweighted Jacknifed. Cluster analysis was preceded by principal component analysis (PCA). Beta-diversity provides a compute of similarity, or dissimilarity, of one microbial composition to another. Beta-diversity is typically calculated on the OTU/ASV/species composition tables directly (after normalization), but can be calculated using abundances at higher taxonomic levels.

While alpha diversity is a measure of the diversity (or complexity) within samples, beta diversity refers to the diversity between samples. This is essentially a measure of how similar or dissimilar the samples are, and is usually represented by a distance matrix which is then used to do Principal Coordinates Analysis (PCOA). The result of this is an ordination plot of multiple dimensions, where each sample is a point and the distance between the points represents the similarity of those samples (closer together = more similar). I used these plots to explore the data and see which variables explain the similarity or dissimilarity of groups of samples (Aitchison and Greenacre, 2002).

\section{Results and Discussion}

To study microbial diversity and population associated with healthy and root rot infected groundnut GG20 plant rhizosphere. Rhizspheric soil samples were collected fom the 5 different sites. From each site collected 10 healthy and 10 root rot infected plants. out of them 20 sample make two samples, one was healthy sample and one was root rot infected sample. Samples were collected from five different type of soil location. So total 10 samples in which 5 healthy plant rhizosphere and 5 rppt rot infected plant rhizosphere soil. Total DNA was extracted direct from all 10 rhizosphere soil using The Power Soil ${ }^{\circledR}$ DNA Isolation Kit. to check the DNA quality performed the gel electrophoresis and got the intect DNA as shown in Figure 1.

Microbial population was studied using the metagenomics approach. after the DNA was extracted, prepare the amplicon using the primer set (16S primer set V2-4-8 16S primer set V3-6,7-9) provided in the $16 \mathrm{~S}^{\mathrm{TM}}$ Metagenomics Kit from thermo fisher. using these primer set prepared the sequencing libraries. sequencing was performed using the Next generation sequencer ION S5 (Iontorrent) of the prepared libraries of all 10 samples. during the sequencing samples were separated by using the IonExpress barcode_001 to Ion Express barcode_010. observations were recorded from the NGS as Figure 2.

Sequencing data was extracted from the the Sequencer. Total $2.5 \mathrm{~GB}$ data in fastQ format. this data included unprocess total reads $1,07,68,140$ with the mean length was $233 \mathrm{bp}$. debarcoding of the sample data gave the following data reads (Table 1).

All sequenced reads were analyzed using the MG-RAST (Metagenomics RAST: metagenomics.anl.gov) server version 3.5, which is an open source service made up of a high-throughput pipeline built for highperformance computing of metagenomes. Screening against a large group of protein and nucleotide databases, MG-RAST allows for the automated assignment of metagenomic sequences to their respective phylogenetic and functional groups for ease of data interpretation, in addition to providing tools 
for comparative metagenomics. The pipeline, implemented in Perl, utilizes a combination of open source components which include the SEED framework (Anderson et al., 2005), Sun Grid Engine, SQLite, and NCBI Blast (Gopalakrishnan et al., 2011).
It is important to check the quality and coverage of the sequencing data using the CLC Genomics Workbench 20.0. analysed the data obtained from the sequencer.

Table.1 Untrimmed raw data representing No. of reads and Mean length present in samples

\begin{tabular}{|c|c|c|c|c|}
\hline S.N. & Sample & Bases in MB & Reads & Mean Read length \\
\hline $\mathbf{1}$ & Healthy-1 & 241.32 & 1022740 & 235 \\
\hline $\mathbf{2}$ & Infected-1 & 507.05 & 1245313 & 236 \\
\hline $\mathbf{3}$ & Healthy-2 & 149.48 & 635376 & 235 \\
\hline $\mathbf{4}$ & Infected-2 & 155.23 & 658089 & 235 \\
\hline $\mathbf{5}$ & Healthy-3 & 275.30 & 1125961 & 244 \\
\hline $\mathbf{6}$ & Infected-3 & 242.10 & 1073193 & 225 \\
\hline $\mathbf{7}$ & Healthy-4 & 264.10 & 1154472 & 228 \\
\hline $\mathbf{8}$ & Infected-4 & 186.61 & 827429 & 225 \\
\hline $\mathbf{9}$ & Healthy-5 & 198.11 & 863458 & 229 \\
\hline $\mathbf{1 0}$ & Infected-5 & 211.36 & 921298 & 229 \\
\hline
\end{tabular}

Table.2 Family, Genus and Species no. of (operational taxonomic unit) OTU presented in all 10 metagenomes

\begin{tabular}{|c|c|c|c|c|}
\hline Sr. No. & Sample & Family OTU & Genus OTU & Species OTU \\
\hline $\mathbf{1}$ & H1 & 336361 & 113208 & 38262 \\
\hline $\mathbf{2}$ & I1 & 817965 & 271234 & 90693 \\
\hline $\mathbf{3}$ & H2 & 201247 & 60035 & 19225 \\
\hline $\mathbf{4}$ & I2 & 215198 & 81489 & 26290 \\
\hline $\mathbf{5}$ & H3 & 478039 & 225603 & 99773 \\
\hline $\mathbf{6}$ & I3 & 420035 & 163045 & 53588 \\
\hline $\mathbf{7}$ & H4 & 481866 & 207818 & 78772 \\
\hline $\mathbf{8}$ & I4 & 325684 & 136267 & 46115 \\
\hline $\mathbf{9}$ & H5 & 366968 & 156751 & 55638 \\
\hline $\mathbf{1 0}$ & I5 & 325480 & 129548 & 41767 \\
\hline
\end{tabular}

Fig.1 Gel of DNA extracted from all 10(H1-H5 and I1-I5) samples

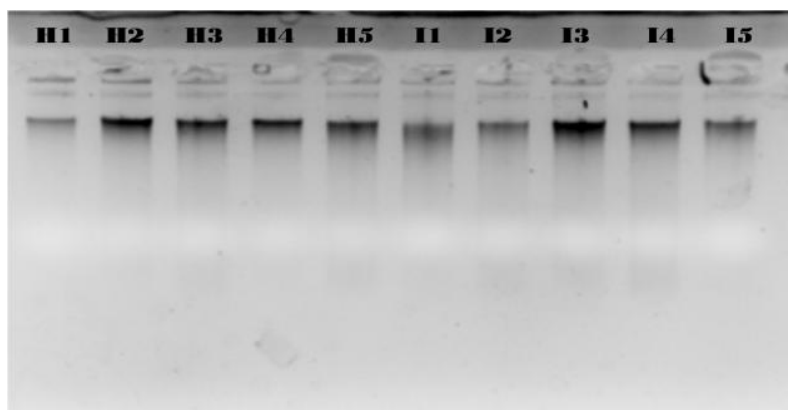


Fig.2 Sequencing run summary representing chip image (Ion 530) and read length
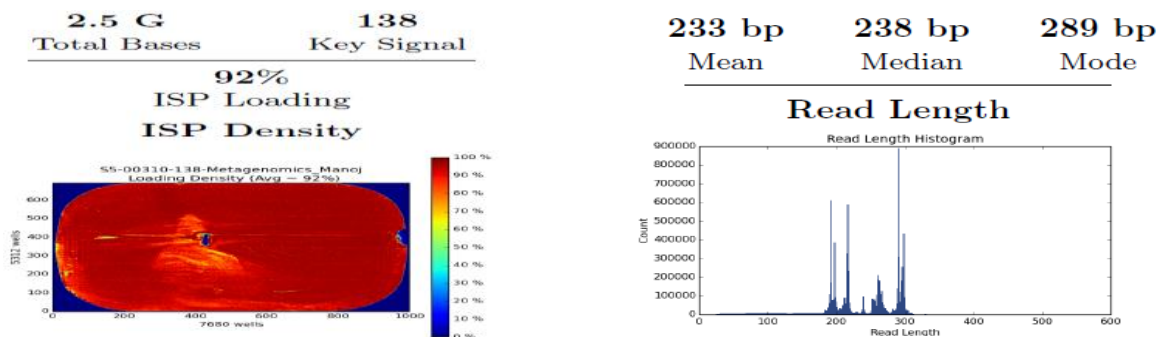

Fig.3 The $\alpha$-diversity of samples representing the species numbers and standard deviation

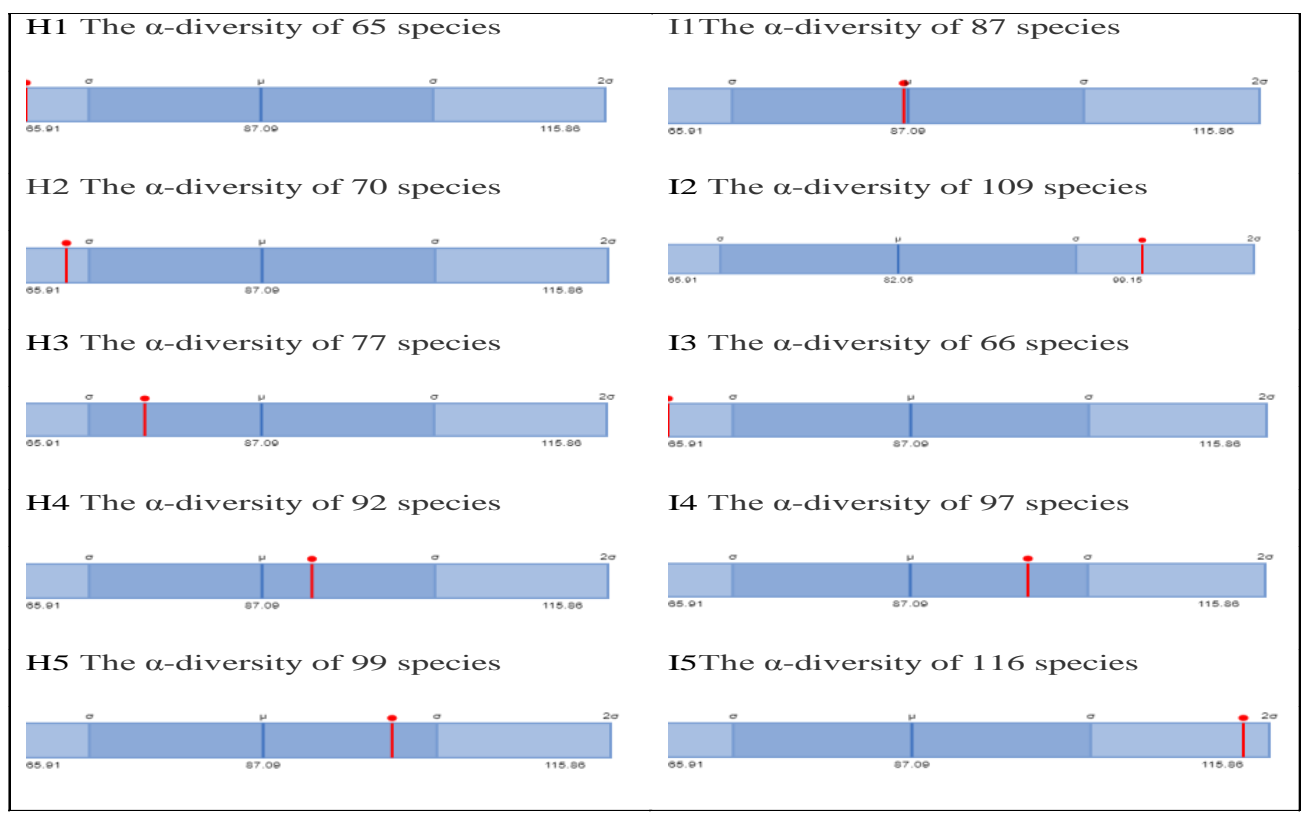

Fig.4 OTU numbers representing Family, Genus and Species of groundnut variety GG-20 Rhizospheric metagenomes of 5 different location of Gujarat at healthy and infection stage. (Xaxis represent sample and $\mathrm{Y}$-axis represent OTU)

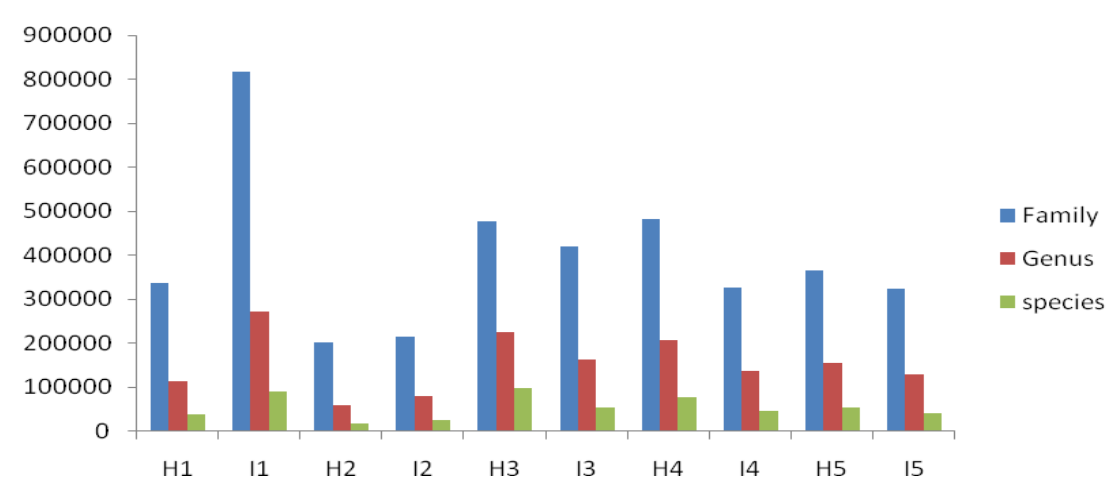


Fig.5 Principle component analysis based on unweighted unifrac $2 d$ reveals sequence based grouping of Family, Genus and species level in A) Healthy H1, H2, H3, H4 and H5. B) Infected I1, I2,I3,I4 and I5 C) Healthy and Infected samples

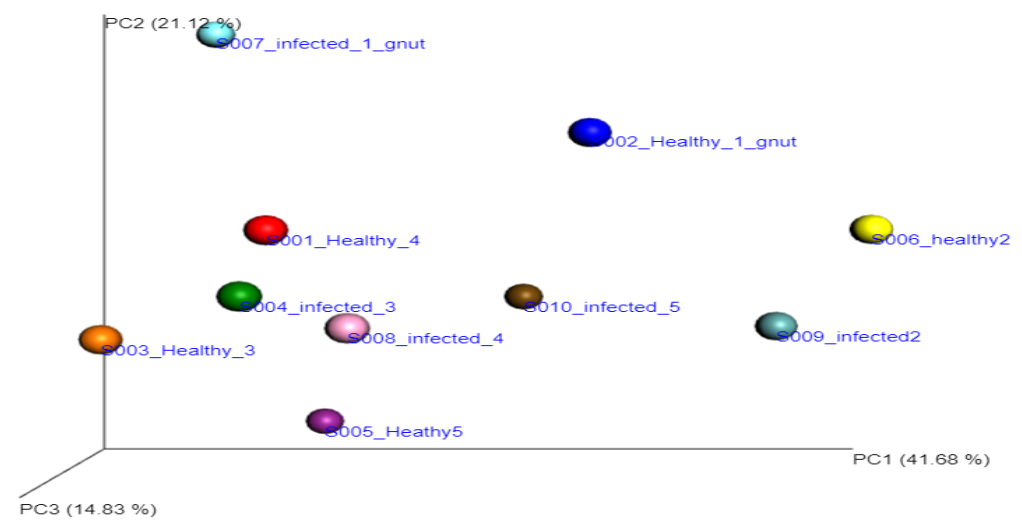

\section{GC Content of sequencing data}

GC content of any sequencing data is specific for strains and the the GC content of a gene region can impact its coverage, with regions having $50-60 \% \mathrm{GC}$ content receiving the highest coverage while regions with high (70$80 \%$ ) or low (30-40\%) GC content having significantly decreased coverage. our result based on CLC analysis all the samples sequencing data had GC content $50-65 \%$ so we can said that our sequencing data have sufficient coverage. The PHERD score of all the samples were between 17-32 and most of the sequences score was 30 . So it was indicated that the quality of data was good and have $99.99 \%$ accuracy.

\section{Bacterial diversity analysis}

\section{Alpha-diversity}

The alpha-diversity of the microbial metagenomes in each site was calculated to assess both species richness and evenness. The below image shows the range of $\alpha$ diversity values in the study 'Groundnut metagenome'. The min, max, and mean values are shown, with the standard deviation ranges ( $\sigma$ and $2 \sigma$ ) in different shades. The $\alpha$-diversity of this metagenome is shown in red. Alpha diversity summarizes the diversity of organisms in a sample with a single number. The $\alpha$-diversity of annotated samples can be estimated from the distribution of the specieslevel annotations. according to result the infected soil sample show more diversity then the healthy soil sample (Figure 3).

\section{Analysis using QIIME pipeline}

Sequence data further processed using the Quantitative Insights Into Microbial Ecology (QIIME), It is an open-source bioinformatics pipeline for performing microbiome analysis from raw DNA sequencing data (McPherson et al., 2018) (Caporaso et al., 2010). from all the 10 metagenomes, no. of valid reads were uploaded in QIIME. the low quality reads were removed before the uploading. second the raw seuences data also uploaded in MGRAST open source pipeline to analyse metagenome (http://api.metagenomics. anl.gov/ api.html). in this software reads were blast against the ribosomal database, genebank data base and converted to OTU (Operational Taxonomic Unit). In MGRAST the OTU analysed up to genus while in QIIME analysed up to the specceies. from the QIIME pipeline we got no. of OTU for 
family, genus and species as Table 2 . We observed that no. of OTU for family, genus and species were different from location to location mens different in $\mathrm{H} 1, \mathrm{H} 2, \mathrm{H} 3, \mathrm{H} 4$ and $\mathrm{H} 5$ samples. while the no. of family OTU high in $\mathrm{H} 4$ samples and low in $\mathrm{H} 2$ samples. no. of genus OTU was found high in $\mathrm{H} 3$ samples and low in $\mathrm{H} 2$ sample. same trend was observed for no. of species OTU (Fugure4).

\section{Beta diversity analysis}

To compute the differences in microbial community composition between samples, in QIIME Bray-Curtis dissimilarity was calculated and visualized in a Principle component analysis (PCA) plot. The pattern of separation between samples indicated distinct microbial community composition between the healthy and infected rhizosphere soil as well as between soil types (Figure 5).

At family, Genus and species level community composition two axes explained more than $60 \%$ of microbial community variance between samples, with samples clearly separated by soil type on the first axis (41.68\% explained variance). The community structure and composition of the plant associated microbiome depends on several factors, such as the soil properties, plant nutritional status, climate, plant genotype, and even the developmental stage of the host plant (Bulgarelli et al., 2015; Pii et al., 2016; Trognitz et al., 2016; Turner, et al., 2013). The microbial composition of healthy and infected samples were distinct.

Present study is that the microbial population was divers from location to location and the status of plant health. the antagonistic microorganisms such as bacteria and fungi are an alternative source for controlling the plant pathogens. some bacterial species form healthy samples have plant growth promoting activities. while some bacterial species have characteristics to suppress the growth of plant pathogen or to protect the plant from the pathogen. microbiome of rhizosphere manipulation will be useful to control the plant disease. microbiome manipulated by adding of the some beneficial microbes into the rhizosphere.

\section{References}

Anderson, I.; Sorokin, A.; Kapatral, V.; Reznik, G.; Bhattacharya, A.; Mikhailova, N.; Burd, H.; Joukov, V.; Kaznadzey, D.; Walunas, T.; Markd'Souza, Larsen, N.;Pusch, G.; Liolios, K.; Grechkin, Y.; Lapidus, A.; Goltsman, E.; Chu, L.; Fonstein, M. and Ivanova, N. 2005. Comparative genome analysis of Bacillus cereus group genomes with Bacillus subtilis. FEMS Microbiology Letter, 250(2): 175-184.

Brussaard, L.; de Ruiter, P.C. and Brown, G.G. 2007. Soil biodiversity for agricultural sustainability. Agriculture, Ecosystems and Environment, 121:233244.

Bulgarelli, D.; Garrido-Oter, R.; Münch, P. C.; Weiman, A.; Dröge, J. and Schulze-Lefert, P. 2015. Structure and function of the bacterial root microbiota in wild and domesticated barley. Cell Host and Microbe, 17(3): 392-403.

Garbeva, P.; van Veen, J.A. and van Elsas, J.D. 2004. Microbial diversity in soil: selection microbial populations by plant and soil type and implications for disease suppressiveness. Annual Review of Phytopathology, 42:243-70.

Garbeva, P.; van Veen, J.A. and van Elsas, J.D. 2004. Microbial diversity in soil: selection microbial populations by plant and soil type and implications for disease suppressiveness. Annual Review of Phytopathology, 42:243-70.

Gopalakrishnan, S.; Humayun, P.; Kiran, B. 
K.; Kannan, I. G. K.; Vidya, M. S.; Deepthi, K. and Rupela, O. 2011. Evaluation of bacteria isolated from rice rhizosphere for biological control of charcoal rot of sorghum caused by Macrophomina phaseolina (Tassi) Goid. World Journal of Microbiology and Biotechnology, 27(6): 1313-1321

Gupta, S. C. and Kolte, S. J. 1981. Cultural characteristics of leaf and root isolates of $M$. phaseolina (Tassi) Goid from groundnut. Indian Journal of Medical Microbiology, 21(4) : 345-346.

Janvier, C.; Villeneuve, F.; Alabouvette, C.; Edel-Hermann, V.; Mateille, T. and Steinberg,C. 2007. Soil health through soil disease suppression: which strategy from descriptors to indicators? Soil Biology and Biochemistry, 39:1-23.

Mendes, R.; Kruijt, M.; de Bruijn, I.; Dekkers, E.; van der Voort, M.; Schneider, J. H.; Piceno, Y. M.; De Santis, T. Z.; Andersen, G. L.; Bakker, P.A. and Raaijmakers, J. M. 2011. Deciphering the rhizosphere microbiome for disease-suppressive bacteria. Science, 332 (6033): $1097-$ 1100

Nannipieri, P.; Ascher, J.; Ceccherini, M.T.; Landi, L.; Pietramellara, G.; Renella, G. and Valori, F. 2008. Effects of root exudates in microbial diversity and activity in rhizosphere soils. Soil Biology, 15: 339-365.

Nannipieri, P.; Ascher, J.; Ceccherini, M.T.; Landi, L.; Pietramellara, G. and Renella, G. 2003. Microbial diversity and soil functions. European Journal of Soil Science, 54:655-670

Trognitz, F.; Hackl, E.; Widhalm, S. and Sessitsch, A. 2016. The role of plant-microbiome interactions in weed establishment and control. FEMS Microbiology Ecology, 92(10): 138

Turner, J.T. and Backman, P.A. 1991. Factors relating to peanut yield increases after seed treatments with Bacillus subtilis Plant Disease, 75:347-353.

Weller, D.M.; Raaijmakers, J.M.; Gardener, B.B. and Thomashow, L.S. 2002. Microbial populations responsible for specific soil suppressiveness to plant pathogens. Annuals Reviews of Phytopathology, 40: 309-48.

Yin, B.; Crowley, D.; Sparovek, G.; De Melo, W.J. and Borneman, J. 2000. Bacterial functional redundancy along a soil reclamation gradient. Applied and Environmental Microbiology, 66:43614365.

\section{How to cite this article:}

Parakhia, M. V. and Golakia, B. A. 2021. Microbiome of Groundnut (Arachis hypogaea L.) Rhizosphere Infected with Macrophomina pheseolina Root Rot. Int.J.Curr.Microbiol.App.Sci. 10(02): 141-149. doi: https://doi.org/10.20546/ijcmas.2021.1002.018 